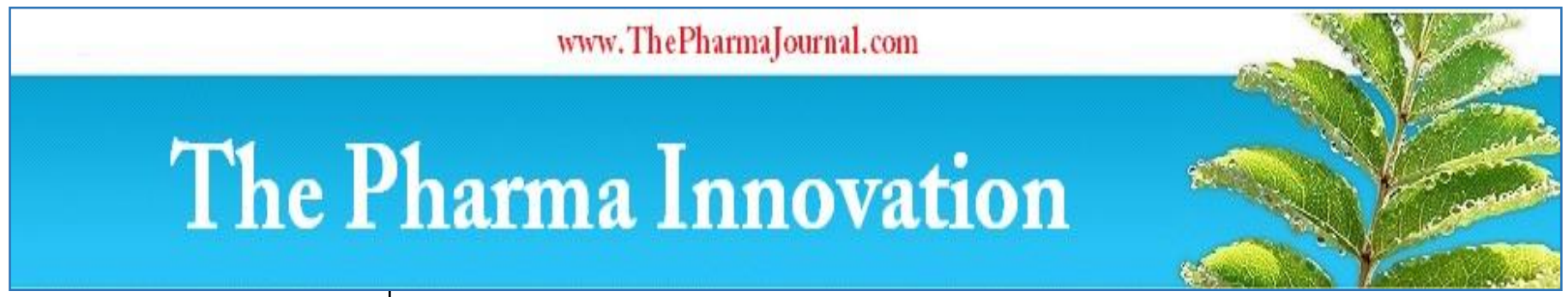

ISSN (E): 2277 - 7695

ISSN (P): 2349-8242

NAAS Rating: $\mathbf{5 . 0 3}$

TPI 2020; 9(7): 173-175

(C) 2020 TPI

www.thepharmajournal.com

Received: 06-05-2020

Accepted: 08-06-2020

Jeevan Ranjan Dash

Department of Veterinary

Pharmacology and Toxicology,

CVSc and AH, OUAT, BBSR,

Bhubaneswar, Odisha, India

Corresponding Author: Jeevan Ranjan Dash

Department of Veterinary

Pharmacology and Toxicology,

CVSc and AH, OUAT, BBSR,

Bhubaneswar, Odisha, India

\title{
TRP channels in uterine pathophysiology- A short review
}

\section{Jeevan Ranjan Dash}

DOI: https://doi.org/10.22271/tpi.2020.v9.i7c.4852

\section{Abstract}

Calcium ions are major upstream signalling molecules to initiate myogenic contraction. TRP (Transient receptor potential) proteins belong to cation channel protein family and are localized in the cell membrane. The most perform of TRPC is to control the inflow of $\mathrm{Ca}^{2+} / \mathrm{Na}^{+}$batting on PKC pathway activation evoked by G-protein-coupled receptors (GPCR). TRPV6, is mostly expressed in pregnant uterus in human, mouse, rat and pig. TRPV6 and TRPC1 are related to embryo implantation. Upregulated TRPC3 urged to play an important role in LPS-induced preterm labor. TRPC1 and TRPM7 are rumoured to related to abnormal uterine fibroid contraction. TRPV4 channels are present within the pregnant and nonpregnant mouse uteri, and their activation by prostaglandin increases myometrial contractility. TRPA1 over activity could also be related to pre-eclampsia and connected cardiovascular diseases. So with growing evidences of upregulation of varied TRP proteins in several pathological conditions of the uterus in human and experimental animals signifies TRP proteins rising as a new therapeutic target in uterine pathophysiology.

Keywords: $\mathrm{Ca}^{+2}$, TRPC, TRPV, TRPM, TRPA, TRPP, Uterus

\section{Introduction}

$\mathrm{Ca}^{+2}$ ion in uterine smooth muscle cells (SMCs) may be a major determinant of uterine contractility (Parkington et al, 1999) ${ }^{[16]}$. Before uterine contractions, cytoplasmic calcium will increase considerably. Recently, varied investigations tested the impact of L-type calcium channels in uterine contraction in mammals as well as human and animals. calcium ions are major upstream signalling molecules to initiate myogenic contraction. The calcium channel proteins are molecular switches in signal transduction pathway and are concerned in cell contraction as well as cell cycle regulation. TRP (Transient receptor potential) proteins belong to cation channel protein family and are localized within the cell membrane. There are regarding twenty eight homologous proteins discovered in mammalian cells until date and that they are in six subfamilies: TRPC, TRPV, TRPM, TRPA, TRPP and TRPML. the most perform of TRPC is to control the inflow of $\mathrm{Ca}^{2+} / \mathrm{Na}^{+}$betting on $\mathrm{PKC}$ pathway activation elicited by the G-protein-coupled receptors (GPCR) (Berridge MJ, 2008) ${ }^{[2]}$. RT-PCR analysis have shown TRPC1, TRPC3, TRPC4, TRPC5, TRPC6 and TRPC7 mRNA whereas western blot analysis and immunolocalization have unconcealed the presence of proteins TRPC1, C3, C4 and C6 in each primary cultured human myometrial SMC (Dalrymple et al., 2002) ${ }^{[7]}$ and immortalized pregnant human myometrial smooth muscle cells (PMH1) (Yang et al., 2002) ${ }^{[21]}$. In rat myometrium, mRNA for TRPC1, TRPC2, TRPC4-C7 were expressed and TRPC4 was the foremost abundant (Babich et al., 2004) ${ }^{[1]}$. So during this minireview an effort is created to insight the role of various TRP channels in several pathophysiological conditions of uterus.

\section{Significance of TRP channel in uterine smooth muscle contraction}

Uterine smooth muscle contraction is mainly regulated by two signalling pathways. one amongst that is calcium signalling. In smooth muscle fibre contraction, the cytosolic $\mathrm{Ca}^{2+}$ is elevated by 2 ways in which. the primary one is by the activation of transmembrane proteins to induce the calcium ion of factor IV. There are 2 varieties of transmembrane calcium channel proteins: calcium transient receptor potential channel (TRPC), and calcium channel delay (long-lasting potential channel, LPC). The activation of G protein-coupled receptor (GPCR) is regulated by the activation of calcium channel proteins. The second one is that the release of intracellular calcium from the endoplasmic reticulum (ER). GPCRs activate PLC (Phospholipase C) and hydrolyze PIP2 (Phosphoinositol diphosphate) within the plasma 
membrane and generate DAG (Dyacyl glycerol) and IP3 (Inositol triphosphate) that binds to IP3 receptors (IP3R) on the endoplasmic reticulum, inflicting calcium release.

\section{TRP Channels in impaired endometrial receptiveness and/or implantation failure.}

TRPV6, a member of TRP family is expressed within the luminal and glandular epithelia in human, mouse, rat and pig endometrium. TRPV6 undergoes cyclic changes throughout the ovarian cycle subject to E2 (estrogen) or progesterone regulation. In humans and mice, the maximal mRNA level of TRPV6 was detected in the oestrus phase, because of predominance of E2 (Lee and Jeung, 2007; rule et al., 2011) [13]. However, in distinction to human and mouse, rat endometrium showed highest TRPV6 mRNA expression at dioestrus (Kim et al., 2006) ${ }^{[12]}$. TRPV6 is additionally highly expressed within the endometrium throughout gestation. In mice, TRPV 6 mRNA was detected ranging from day seven of gestation and peaked at mid-gestation (Lee and Jeung, 2007) ${ }^{[13]}$. In rats and pigs, the maximal mRNA level of TRPV6 was detected at implantation (Choi et al., 2009) ${ }^{[5]}$. TRPC1, a member of the TRP family, has been reportable to be expressed in ESCs (Endometrial smooth muscle cells). Kawarabayashi et al. (2012) [11] incontestable that the E2/progesterone-induced decidualization in vitro was in the course of up-regulation of TRPC1 and will increase in TRPC1-mediated SOC activity in human ESCs (hESCs).

\section{TRP channels and pre-term labour}

In term labor women TRPC3 was detected in higher intensity than in term non-labor woman and in a lot of beyond in unpregnant women (Dalrymple et al, 2003) ${ }^{[8]}$. Senadheera $e t$ al (2013) ${ }^{[17]}$ incontestable that TRPC3 was vital in control uterine contraction in rats, with the mechanism increased in gestation. TRPC3, L-type Cav1.2, T-type Cav3.1, and Cav3.2 localized within the uterine radial artery smooth muscle act synergistically (Senadheera et al, 2013) ${ }^{[17]}$. A study by Zheng et al., $2016^{[22]}$ recommended that upregulated TRPC3 might play a crucial role in LPS-induced preterm delivery.

\section{TRP channels and uterine cancer}

Uterine fibroid is that the commonest nonmalignant tumor of feminine reproductive organs with a clinical incidence of $20 \%-40 \%$ (Ciarmela et al., 2011) ${ }^{[6]}$. The common symptoms related to it embrace abnormal uterine hemorrhage, infertility, and pelvic mass which can cause a heavy threat to women's health and quality of life. Reports say TRPC1 and TRPM7 calcium channel proteins are related to abnormal uterine fibroid contraction. Abnormal TPRC expressions are exhibited in numerous human neoplasm tissues. several studies show that TRPC proteins are related to the proliferation of breast cancer (Ouadid-Ahidouch et al., 2012) ${ }^{[15]}$, prostate cancer (Vanden Abeele et al., 2003) ${ }^{[19]}$, brain tumor cell proliferation (Bomben et al., 2010) ${ }^{[3]}$ and brain tumor cell migration (Bomben et al., 2011) ${ }^{[4]}$. It's been urged that the upregulation of TRPC1 expression enhance intracellular $\mathrm{Ca}^{2+}$ concentration, promoted cell proliferation and suppressed programmed cell death. Meanwhile, cell migration may be a vital step of tumor metastasis and invasion. during this method, TRPC 1 plays a really necessary role by increasing the $\mathrm{Ca} 2+$ gradient and affecting cell migration (Fabian et al., 2008) ${ }^{[10]}$. TRPM7 (Transient receptor potential cation channel, subfamily $\mathrm{M}$, member 7) may be a recently discovered dual function protein acting as an ion channel and protein kinase concerned in uterine fibrosis (Fabian et al., 2008) ${ }^{[10]}$.

\section{TRP channel and pre-eclampsia}

Preeclampsia/eclampsia/hypertension in gestation may be a perinatal/reproductive condition which will considerably increase the danger of death for a pregnant or early postpartum woman and might increase the danger of prematureness and low birth weight for infants. The condition happens in $5-8 \%$ of pregnancies within the U.S. and internationally. many members of an outsized family of nonselective cation entry channels, e.g., transient receptor potential (TRP) canonical (TRPC), vanilloid (TRPV), and melastatin (TRPM) channels, are related to the event of cardiovascular diseases and cardiovascular disease. TRPV4 might regulate the vascular tone of rat carotid artery through associate degree attenuated NO pathway and stimulation of the discharge of contractile prostanoids in the DOCA hypertensive rats (Dash et al, 2019) ${ }^{\text {[9] }}$. Thus, disruption of TRP channel expression or perform might account for the determined increased cardiovascular risk in metabolic syndrome patients. TRPV4 channels are present within the pregnant and nonpregnant mouse uteri, and their activation by endogenous ligands like autacoid will increase myometrial contractility. Thus, the TRPV4 channel are often a crucial target in reducing myometrial contractility in preterm labor (Singh et al., 2015) ${ }^{[18]}$. Pre-eclampsia might involve overactivity of the TRPA1 channel. it's investigated that TRPV1 regulates adipogenesis and inflammation in fat tissues, whereas TRPC3, TRPC5, TRPC6, TRPV1, and TRPM7 are concerned in constriction and regulation of blood pressure (Liu et al., 2008) ${ }^{[14]}$.

\section{Conclusion}

With growing evidences of upregulation of varied TRP proteins in numerous pathological conditions of the uterus in human and experimental animals signifies TRP proteins rising as a brand-new therapeutic target in female internal reproductive organ pathophysiology.

\section{Conflict of interest- none}

\section{References}

1. Babich LG, Ku CY, Young HW, Huang H, Blackburn MR, Sanborn BM et al. Expression of capacitative calcium TrpC proteins in rat myometrium during pregnancy. Biol Reprod. 2004; 70: 919-924.

2. Berridge MJ. Smooth muscle cell calcium activation mechanisms. J Physiol. 2008; 586:5047-5061.

3. Bomben VC, Sontheimer H. Disruption of transient receptor potential canonical channel 1 causes incomplete cytokinesis and slows the growth of human malignant gliomas. Glia. 2010; 58:1145-1156.

4. Bomben VC, Turner KL, Barclay TTC, Sontheimer H. Transient receptor potential canonical channels are essential for chemotactic migration of human malignant gliomas. J Cell Physiol. 2011; 226:1879-1888.

5. Choi Y, Seo H, Kim M, Ka H. Dynamic expression of calcium-regulatory molecules, TRPV6 and S100G, in the uterine endometrium during pregnancy in pigs. Biol Reprod. 2009; 81:1122-1130.

6. Ciarmela P, Islam MS, Reis FM, Gray PC, Bloise E, Petraglia $\mathrm{F}$ et al. Growth factors and myometrium: biological effects in uterine fibroid and possible clinical 
implications. Hum Reprod Update. 2011; 17:772-790.

7. Dalrymple A, Slater DM, Beech D, Poston L, Tribe RM. Molecular identification and localization of Trp homologues, putative calcium channels, in pregnant human uterus. Mol Hum Reprod. 2002; 8:946-951.

8. Dalrymple A, Slater DM, Poston L, Tribe RM. Physiological induction of transient receptor potential canonical proteins, calcium entry channels, in human myometrium: influence of pregnancy, labor, and interleukin-1. J Clin Endocrinol Metab. 2003; 89:12911300.

9. Dash JR, Mishra SK, Parida S, Singh TU, Choudhury S, Muniyappa $\mathrm{K}$ et al. TRPV4 activation in rat carotid artery in DOCA hypertension involves eNOS and endothelium-derived contractile factor (EDCF). Clin Exp Hypertens. 2019; 41(6):564-570.

10. Fabian A, Fortmann T, Dieterich P, Riethmüller C, Schön $\mathrm{P}$, Mally $\mathrm{S}$ et al. TRPC1 channels regulate directionality of migrating cells. Pflugers Arch. 2008; 457:475-484.

11. Kawarabayashi Y, Hai L, Honda A, Horiuchi S, Tsujioka $\mathrm{H}$, Ichikawa $\mathrm{J}$ et al. Critical role of TRPC1-mediated $\mathrm{Ca}^{(2)(+)}$ entry in decidualization of human endometrial stromal cells, Mol Endocrinol. 2012; 26:846-858.

12. Kim HJ, Lee GS, Ji YK, Choi KC, Jeung EB. Differential expression of uterine calcium transporter 1 and plasma membrane $\mathrm{Ca}^{2+}$ ATPase $1 \mathrm{~b}$ during rat estrous cycle. Am J Physiol Endocrinol Metab. 2006; 291:E234-E241.

13. Lee GS, Jeung EB. Uterine TRPV6 expression during the estrous cycle and pregnancy in a mouse model, Am J Physiol Endocrinol Metab. 2007; 293:E132-E138.

14. Liu D, Zhu Z, Tepel M. The Role of Transient Receptor Potential Channels in Metabolic Syndrome. Hypertens Res. 2008; 31:1989-1995.

15. Ouadid-Ahidouch H, Dhennin-Duthille I, Gautier M, Sevestre H, Ahidouch A. TRP calcium channel and breast cancer: expression, role and correlation with clinical parameters. Bull Cancer. 2012; 99:655-664.

16. Parkington HC, Tonta MA, Davies NK, Brennecke SP, Coleman HA. Hyperpolarization and slowing of the rate of contraction in human uterus in pregnancy by prostaglandins $E_{2}$ and $F_{2 a}$ : involvement of the $\mathrm{Na}^{+}$ pump. J Physiol. 1999; 514:229-243.

17. Senadheera S, Bertrand PP, Grayson TH, Leader L, Tare M, Murphy TV et al. Enhanced contractility in pregnancy is associated with augmented TRPC3, L-type, and T-type voltage-dependent calcium channel function in rat uterine radial artery. Am J Physiol Regul Integr Comp Physiol. 2013; 305:R917-926.

18. Singh V, Ram M, Kandasamy K, Thangamalai R, Choudhary S, Dash JR et al. Molecular and functional characterization of TRPV4 channels in pregnant and nonpregnant mouse uterus. Life sciences. 2015; 122:5158.

19. Vanden Abeele F, Shuba Y, Roudbaraki M, Lemonnier L, Vanoverberghe K, Mariot $\mathrm{P}$ et al. Store-operated $\mathrm{Ca}^{2+}$ channels in prostate cancer epithelial cells: function, regulation, and role in carcinogenesis. Cell Calcium. 2003; 33:357-373.

20. Yang H, Choi KC, Hyun SH, Jeung EB. Coexpression and estrogen-mediated regulation of TRPV6 and PMCA1 in the human endometrium during the menstrual cycle. Mol Reprod Dev. 2011; 78:274-282.

21. Yang M, Gupta A, Shlykov SG, Corrigan R, Tsujimoto $\mathrm{S}$, Sanborn BM et al. Multiple Trp isoforms implicated in capacitative calcium entry are expressed in human pregnant myometrium and myometrial cells. Biol Reprod. 2002; 67:988-994.

22. Zheng D, Zhang L, Na Q et al. Enhanced expression of transient receptor potential channel 3 in uterine smooth muscle tissues of lipopolysaccharide-induced preterm delivery mice. Iran J Basic Med Sci. 2016; 19(5):567572 . 